\title{
Conventional Borescope in the Evaluation and Management of Nasal Schistosomiasis in a Bullock
}

\author{
S. Yogeshpriya ${ }^{1 *}$, P. Selvaraj ${ }^{2}$, M. Saravanan ${ }^{1}$, P. K. Ramkumar ${ }^{1}$ and S. Asmitha ${ }^{1}$ \\ ${ }^{1}$ Department of Veterinary Medicine, Veterinary College and Research Institute, \\ Orathanadu, India \\ ${ }^{2}$ Department of Veterinary Clinical Medicine, Madras Veterinary College, Chennai, India \\ *Corresponding author
}

\section{A B S T R A C T}

\section{Keywords \\ Borescope, Snoring diseases, Lithium antimony tartarate, Bullock \\ Article Info \\ Accepted: \\ 20 December 2020 Available Online: 10 January 2021}

Diagnosis of mucosal abnormalities in nasal cavity is efficiently by endoscopy, which is sophisticated and costly equipment available in referral units only. Conventional Borescope could be used in the diagnosis of nasal Schistosomiasis in field level. Such a case, an application of low cost conventional Borescope was used in the diagnosis of nasal Schistosomiasis in a bullock. A 15 year old Kankeyam bullock was presented to theLarge Animal Medicine Referral Clinic with the history of anorexia, bilateral nasal discharge and snoring since 15 days. Borescopic examination revealed nodular growth in the nasal passage, further confirmed by nasal washing examination, as Schistosoma nasale. The animal was treated with Lithium antimony tartarate along with supportive care.

\section{Introduction}

Neglected tropical diseases (NTDs), are a group of diseases that occur under tropical and sub-tropical climate conditions and are intimately linked to poverty. Among NTDs, Water-associated infectious diseases can be broadly classified as water-borne, waterbased, water-dispersed, water-related and water-washed. The best known classical example of water-based infectious disease is schistosomiasis caused by a human contact with water infested with the larva of some parasitic worms known as cercariae. In humans, Schistosomiasis ranks second after malaria for parasitic diseases in terms of mortality and morbidity (Karunamoorthi et al., 2018).

Schistosomiasis is recognized as a tropical disease of considerable public health importance, but domestic livestock infections due to Schistosoma japonicum, S. bovis, S. mattheei, S.nasale and S. curassoni are often overlooked causes of significant animal morbidity and mortality in Asia and Africa. Schistosoma nasale, inhabits the blood vessels of the nasal mucosa and causes 
disease commonly known as 'nasal schistosomiasis' or 'snoring disease' in animals especially cattle (You et al., 2018).

Nasal Schistosomiasis in cattle was confirmed by microscopical examination of nasal wash/discharge/scrapings (Muraleedharan, 2018). Rhinoscopic examination plays vital role in the diagnosis of lesions in nasal passage and to obtain mucosal biopsy for routine histopathology (Ponnuswamy et al., 2016). However, rhinoscopic examination routinely performed in referral units only. In field condition application of Rhinoscopy is very challenging because its costly equipment and moving frequently place to place will affects the quality of the equipment, hence the Rhinoscopic examination in field condition is not possible. Hence conventional borescope could replace the limitation of application of Rhinoscopy in the field condition. Based on this the present case describe about application of conventional borescope in Schistosoma nasale and their medical management in a bullock.

\section{Materials and Methods}

Clinical case is a 15 year old non-descriptive bull presented in Large Animal Medicine Unit of VCRI, Orathanadu during the month of July 2018 with a history of anorexia, nasal discharge, inspiratory dyspneoa with snoring since 15 days. Animal was subjected to routine physical examination and haematobiochemical analysis. Faecal sample was collected in a clean polythene bags container and direct smear and sedimentation techniques employed for faecal analysis. Direct examination of nasal mucosa showed a small peanut size granuloma like growth (Fig.1).

Commercially available Flexible, waterproof inspection Borescope camera LED light (8 $\mathrm{mm}$ diameter) with 1 meter length was used for the present case to examine the nasal cavity. Borescope easily connected with laptop and Android phone and moreover its cost effective and easily to carry any ware (Fig.2). Before examination Borescope was cleaned with antiseptic solution. Borescopic procedure was performed standing posture without any sedation (Fig.3). Borescope was connected with android device and image was stored.

Nasal scrapings and washings were collected in normal saline solution and analyzed for fungal infection. The direct microscopic examination of nasal washings/scrapings revealed boomerang/palanquin shaped egg with terminal spine and fully developed miracidium inside (Fig.4). On faecal examination, no parasitic eggs could be detected.

The case was diagnosed as Nasal schistosomiasis and the size of the lesion were ruled out by using hand held borescope. The case was differentially diagnosed with Nasal granuloma, Allergic condition (Eosinophilic Allergic Rhinitis), Cystic enlargement of ventral nasal conchae, Rhinosporidiosis, Nasal Schistosomiasis.

Animal was treated with Injection Lithium antimony tartarate @ 20ml intramuscularly along with Injection Chlorpheniramine maleate $(0.5 \mathrm{mg} / \mathrm{kg}$ bwt $\mathrm{i} / \mathrm{m})$ in weekly interval for 3 weeks. Injection Streptopenicillin $(20,000 \mathrm{IU} / \mathrm{kg}$ bwt, i/m) and Injection Meloxicam (0.5 mg / kg bwt, i/m). The animal was administered with Suspension Triclabendazole @ 20mg/kg b.wt orally. Hence In support of our findings, treating animals with these specific signs with lithium antimony given fruitful results.Follow up treatment was advised to the owner and reviewed after three weeks for the regression of nodules. 


\section{Results and Discussion}

Attempts were made on the epizootiology of nasal schistosomiasis to obtain thorough relevant information on this disease. Different studies showed occurrence of $S$. nasale in Andhra Pradesh, Tamil Nadu, West Bengal, Assam, Bihar, Orissa and Maharashtra.
Symptoms like anorectic observed inthis case may be due to irritation and inflammation caused to the nasal mucosa. Even though many other diseases will occur with same symptoms, infestation with $S$. nasale should be kept in mind while diagnosing the cause of disease, especially in highly irrigated Cauvery Delta Region (CDR).

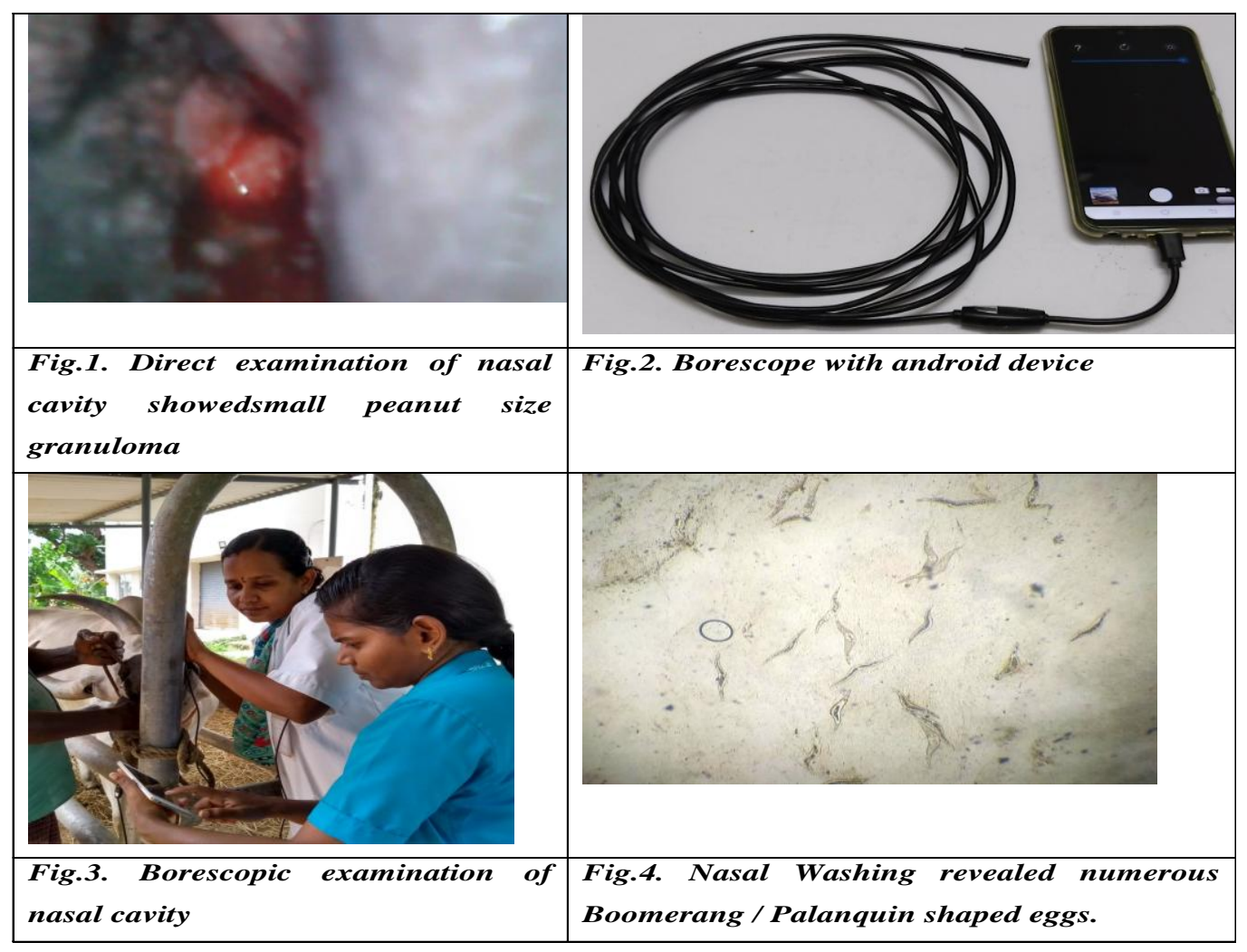

Most of the field veterinarians would examine nasal washing only in case of observing specific sign "snoring" in bovines. However, which only occur when the disease is severe, developed to polyps and obstructing the air way. In such cases, cost effective, easy and quick examination of nodules by borescope is advantageable than rhinoscopy examination except for biopsy collection. In the present case, traditional flexible video boroscope was used. It is an optical instrument designed to assist visual inspection of narrow, difficult-toreach cavities, end of the insertion tube consisting of miniature video camera, light source at the end of the flexible tube. An internal image of the illuminated object is formed by the objective lens and magnified by the eyepiece which presents it to the viewer's eye. Photo or videographic device can be externally linked to the Rigid or flexible borescopes. Since boroscope used in this case helped in the field level and reduced time consumption to visualize the nodules. Whereas rhinoscopic examination of nasal cavity taken lot of time and results in a heavy economically loss to owners because of drop on production.

Animals showed recovery after the administration ofLithium antimony tartarate. 
Antimony attached itself to sulphur atoms in trypanothione reductase which was used by the Schistosoma nasale. Hence, the administration of Lithium antimony tartarate might be a choice of the treatment (Vaidyanathan, 1949) along with antihelminthics in this case. Owner was advised to not allow the cow to graze near the water area and advised to do regular deworming on the interval of six months.Both animals and humans should be screened for Schistosomiasis in water logging areas and should create Public awareness.

In conclusion, schistosomiasis should be taken into consideration as a one of the major limiting factor to livestock productivity in the Cauvery Delta Region (CDR); hence any attempt towards animal disease control strategy in CDR must include it in the priority list. Those borescopic investigation of narrow cavity needs further clinical studies in more number of animals for the validation of conventional borescope in the veterinary practice. Hence, to assess efficacy of therapy, boroscope can be used as a clinical tool for field practitioners.

\section{References}

Karunamoorthi K, Almalki MJ, Ghailan KY.Schistosomiasis: A neglected tropical disease of poverty: A call for intersectoral mitigation strategies for better health. J Health Res Rev. 2018;5:1-12.

Muraleedharan, K. (2018). Nasal
Schistosomosis In Indian Livestock Retrospective for Five Decades. International Journal of Food, Agriculture and Veterinary Sciences. 8 (3).1-14.

Ponnuswamy, K.K.,Sivaraman, S., and Venkatesakumar, E., Sivaseelan, S. and Vijayakumar, G.(2016). Rhinoscopic diagnosis of nasal schistosomiasis and its medical management in a cow. Indian Veterinary Journal. 93. 33-35.

Vaidyanathan, S.N. (1949). Schistosoma spindalis in a cow. Treatment with Anthiomaline. Indian veterinary journal. 26:225-228

Yogeshpriya S, Saravanan M, Jayalakshmi M, Veeraselvam S, Krishnakumar S and Selvaraj P (2017). Nasal schistosomiasis in cattle- A clinical case report. International journal of science, environment and technology, 6:2, 10711074.

Yogeshpriya, S., Veeraselvam, M., Jayalakshmi, K., Krishnakumar, S and Selvaraj,P. (2018). Epidemiology and Clinical Features of Naturally Occurring Nasal Schistosomiasis in Cattle from Delta Region of Tamilnadu. Indian Vet. J.,95 (03) : 81 - 82

You, H., Cai, P., Tebeje, B. M., Li, Y., \& McManus, D. P. (2018). Schistosome Vaccines for Domestic Animals. Tropical medicine and infectious disease, $\quad 3(2), \quad 68$. https://doi.org/10.3390/tropicalmed3020 068.

\section{How to cite this article:}

Yogeshpriya, S., P. Selvaraj, M. Saravanan, P. K. Ramkumar and Asmitha, S. 2021. Conventional Borescope in the Evaluation and Management of Nasal Schistosomiasis in a Bullock. Int.J.Curr.Microbiol.App.Sci. 10(01): 3024-3027. doi: https://doi.org/10.20546/ijcmas.2021.1001.351 